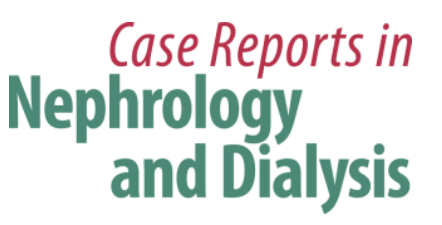

Case Rep Nephrol Dial 2015;5:204-212

DOI: $10.1159 / 000441715$

Publisnea onine: vecember 15, 2015

\title{
Macrophage Infiltration into the Glomeruli in Lipoprotein Glomerulopathy
}

\author{
Satoshi Takasaki ${ }^{a} \quad K^{2}$ nihiko Maeda ${ }^{b}$ Kensuke Joh ${ }^{c}$ Shu Yamakage ${ }^{d}$ \\ Sachiko Fukase ${ }^{d}$ Toshiyuki Takahashi ${ }^{d}$ Masayuki Suzuki ${ }^{d}$ \\ Akira Matsunaga $^{e}$ Takao Saito ${ }^{f}$ \\ ${ }^{a}$ Department of Internal Medicine, Saiseikai Yamagata Saisei Hospital, and ${ }^{\mathrm{b}}$ Department of \\ Nursing, Faculty of Health Science, Yamagata Prefectural University of Health Sciences, \\ Yamagata, 'Department of Pathology, Tohoku University Graduate School of Medicine, \\ Sendai, dDepartment of Internal Medicine, Yamagata Prefectural Central Hospital, \\ Yamagata, ${ }^{\mathrm{e}}$ Department of Laboratory Medicine, Fukuoka University School of Medicine, \\ and ${ }^{f}$ Department of Internal Medicine, Fukuoka University School of Medicine, \\ Fukuoka, Japan
}

\section{Key Words}

apoE2 · apoE Tokyo/Maebashi · Form cell · Lipoprotein glomerulopathy · Macrophage

\begin{abstract}
Lipoprotein glomerulopathy (LPG) is characterized by histopathological features showing intra-glomerular lipoprotein thrombi and type III hyperlipoproteinemia (HLP), with heterozygote mutation of apolipoprotein (apo) E gene. On the other hand, as another renal lipidosis with type III HLP, apoE2 homozygote-related glomerulopathy (apoE2-GN) showing foamy macrophages has been reported. The case of a 25 -year-old man who had LPG by clinical behavior and gene analysis, but demonstrated atypical histopathological features with a substantial amount of foamy macrophage infiltration in the glomeruli, is presented. The combination of alleles for apoE Tokyo/Maebashi and classical apoE2 (Arg158Cys) was inferred to be the leading cause of the unique renal pathology with lipoprotein thrombi and foamy macrophages. In addition, foamy macrophages infiltrated some part of the apoE-positive region within the glomerulus, but did not exist in lipoprotein thrombi despite apoE positivity, suggesting that properties of apoE are crucial in the development of LPG rather than macrophage function. This case provides important information related to the pathogenesis of LPG and apoE2-GN.

(C) 2015 The Author(s)

Published by S. Karger AG, Basel
\end{abstract}

Satoshi Takasaki, MD

Saiseikai Yamagata Saisei Hospital

79-1 Oki-machi, Yamagata 990-8545 (Japan)

E-Mail stakasak@yahoo.co.jp 


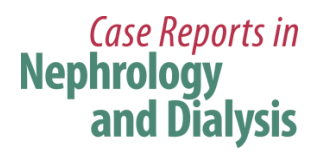

\begin{tabular}{l|l}
\hline Case Rep Nephrol Dial 2015;5:204-212 \\
\hline DOI: 10.1159/000441715 & $\begin{array}{l}\text { ○ 2015 The Author(s). Published by S. Karger AG, Basel } \\
\text { www.karger.com/cnd }\end{array}$ \\
\hline
\end{tabular}

Takasaki et al.: Macrophage Infiltration into the Glomeruli in Lipoprotein Glomerulopathy

\section{Introduction}

Lipoprotein glomerulopathy (LPG) is a rare inherited disease first reported in 1989 by Saito et al. [1]. LPG is sometimes associated with nephrotic syndrome and is characterized by histopathological features of lipoprotein thrombi in extremely dilated glomerular capillaries [2]. Type III hyperlipoproteinemia (HLP) with specific mutations of apolipoprotein (apo) E gene is another diagnostic check point. Histologic alterations other than lipoprotein thrombus found in renal lipidosis, such as infiltration of foamy macrophages, mesangial hypercellularity and exudative lesions, are rarely seen in the glomeruli of typical LPG.

In the present report, a case that was regarded as a category of LPG by clinical behavior and the gene analysis, but demonstrated atypical histopathological features with foamy macrophage infiltration, is described. The combination of alleles for apoE gene Tokyo/Maebashi and classical apoE2 (Arg158Cys) in this case was inferred to be the leading cause of the unique renal pathology with lipoprotein thrombi and foamy macrophages.

\section{Case Report}

A 25-year-old Japanese man was admitted to our hospital because of proteinuria and general edema. Proteinuria and high blood pressure had been detected at a routine medical check-up 3 years earlier. His past history was otherwise unremarkable. Although his mother suffered from nephrolithiasis, there was no family history of renal diseases such as nephrotic syndrome and renal failure. At the first visit to our hospital, his height was $172 \mathrm{~cm}$, weight was $76.2 \mathrm{~kg}$, blood pressure was $170 / 120 \mathrm{~mm} \mathrm{Hg}$, and pulse rate was $91 \mathrm{bpm}$. Physical examination revealed no particular abnormalities other than pitting edema of the lower extremities. He had neither corneal opacities nor xanthomata. Laboratory findings were as follows: white blood cell count $7,700 / \mu \mathrm{l}$, (neutrophils $88.0 \%$, lymphocytes $4.0 \%$, eosinophils 4.0\%), hemoglobin $11.6 \mathrm{~g} / \mathrm{dl}$, platelet count $295,000 / \mu \mathrm{l}$, total protein $4.0 \mathrm{~g} / \mathrm{dl}$, albumin 1.6 $\mathrm{g} / \mathrm{dl}$, hemoglobin A1c 5.3\%, serum creatinine $1.36 \mathrm{mg} / \mathrm{dl}$, total cholesterol $887 \mathrm{mg} / \mathrm{dl}$, triglycerides $553 \mathrm{mg} / \mathrm{dl}$, and HDL-cholesterol $45 \mathrm{mg} / \mathrm{dl}$. Liver function and total complement were within normal ranges. Serum IgG, IgA, and IgM were 213, 116, and $235 \mathrm{mg} / \mathrm{dl}$, respectively. Anti-nuclear antibody and anti-neutrophil cytoplasmic antibody were negative. The serum apoE level was $14.6 \mathrm{mg} / \mathrm{dl}$ (normal range: $2.7-4.3 \mathrm{mg} / \mathrm{dl}$ ). Urine protein excretion was $12.9 \mathrm{~g} / \mathrm{g} \mathrm{Cr}$, and urine sediment RBC was 20-29/hpf.

\section{Clinical Course}

Initial treatment consisted of high-dose corticosteroid for nephrotic syndrome. However, corticosteroid treatment was withdrawn because the first biopsy suggested LPG. After antihyperlipidemic treatment with atorvastatin, the serum cholesterol and triglyceride levels decreased substantially, but urinary protein excretion continued in the nephrotic range. Thereafter, the use of probucol and bezafibrate brought a significant decrease in urine protein excretion $(0.3 \mathrm{~g} / \mathrm{g} \mathrm{Cr})$. Though serum cholesterol and triglyceride levels were reduced, the serum apoE level did not change with lipid-lowering therapy including bezafibrate (before $14.6 \mathrm{mg} / \mathrm{dl}$, after $12.4 \mathrm{mg} / \mathrm{dl}$ ).

\section{Pathological Findings}

First Biopsy (January 2013). The light microscopy specimens contained 40 glomeruli, 7 and 2 of which showed global and segmental sclerosis, respectively. Most glomeruli showed not only typical lipoprotein thrombi filled in the dilated capillaries, but also many infiltrating 


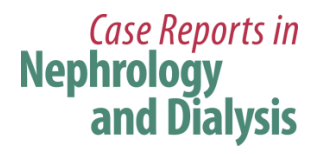

\begin{tabular}{l|l}
\hline Case Rep Nephrol Dial 2015;5:204-212 \\
\hline DOI: 10.1159/000441715 & $\begin{array}{l}\text { (c) 2015 The Author(s). Published by S. Karger AG, Basel } \\
\text { www.karger.com/cnd }\end{array}$ \\
\hline
\end{tabular}

Takasaki et al.: Macrophage Infiltration into the Glomeruli in Lipoprotein Glomerulopathy

foam cells (fig. 1a). Diffuse/segmental mesangial cell proliferation and exudative lesions were also observed. Immunohistochemical studies using serial sections demonstrated strong staining for both apoB and apoE within the dilated capillary lumen filled with lipoprotein thrombi (fig. 1b, c). In addition, positivity for apoE was also observed at sites other than lipoprotein thrombi (fig. 1c). Intriguingly, CD68+ foam cells infiltrated in the apoE-positive parts other than lipoprotein thrombi (fig. 1d). Electron micrographs for the glomerulus showed intra-capillary lipoprotein thrombus-like substances (fig. 2a) and foamy changes (fig. 2b).

Second Biopsy (September 2013). The light microscopy specimens contained 23 glomeruli. Although lipoprotein thrombi and foam cells were still observed, their number was much lower than on the first biopsy (data not shown). Immunofluorescence examinations showed only non-specific IgM deposition along the capillary wall (data not shown).

\section{Phenotype, Genotype, and DNA Sequence Analysis of apoE}

Serum apoE phenotypes were analyzed using isoelectric focusing polyacrylamide gel electrophoresis (IEF), as previously described [3, 4]. As compared to apoE3/3 of control wild type, the patient had alleles apoE1/2 (fig. 3a).

The apoE genotype was determined by restriction fragment length polymorphism (RFLP) analysis as described previously [3, 4]. Genomic DNA was amplified by polymerase chain reaction (PCR) using oligonucleotide primers, sense 5'-ACGCGGGCACGGCTGTCCAAGGA-3' and antisense $5^{\prime}$-TCGCGGGCCCCGGCCTGGTACAC- $3^{\prime}$. The PCR products were digested with the restriction enzyme HhaI. In addition to the fragments characteristic of genotype $\varepsilon 2$ (91-, 78-, and 36-bp fragments), a 30-bp fragment which was not seen in genotype $\varepsilon 2$ and an around 60 -bp fragment which was not detected in any of the common genotype $\varepsilon 2$, $\varepsilon 3$, or $\varepsilon 4$ were identified (fig. 3b).

Sequencing of apoE DNA was performed as follows. Three fragments of genomic DNA containing all coding sequence of mature apo $\mathrm{E}$ were amplified by polymerase chain reaction with the following primers for apo E gene: $5^{\prime}$-GCTTTCCAAGTGATTAAACCGACT-3' and $5^{\prime}$ AGAGCTAAAGCCAGGAGTCAG- $3^{\prime}$ for exon 3 , and $5^{\prime}$-CCTCTTGGGTCTCTCTGGCT- $3^{\prime}$ and $5^{\prime}$ CTGCTCCTTCACCTCGTCCA-3', 5'-GCAGTACCGCGGCGAGGTGCAGG-3' and 5'-GATCGTGCCACTGCACTCTA-3' for exon 4. The amplified DNA fragments were purified by PCR purification Kit (Qiagen, Germany) and directly sequenced with Genetic Analyzer 3130xl DNA sequencer (Thermo Fisher, USA) by using a BigDye Terminator Cycle Sequencing Kit (Thermo Fisher). A heterozygous deletion of $9 \mathrm{bp}$ was identified in codon 141 to codon 143 of the apoE gene (fig. 3c). This mutation was consistent with apoE Tokyo/Maebashi mutation in LPG patients, as previously reported [5, 6]. Furthermore, the patient also had heterogeneous apoc2 (Arg158Cys) (fig. 3d). Because $\varepsilon 2$ corresponds to apoE phenotype E2, apoE Tokyo/Maebashi mutation seemed to be expressed as the apoE phenotype E1 by IEF (fig. 3a). This phenomenon was consistent with a previous report [5]. These findings indicate that this patient was a compound heterozygote for apoE Tokyo/Maebashi and apoE2. Written informed consent was obtained from the patient before commencement of the phenotype and gene analysis of apoE.

\section{Discussion}

LPG is a rare inherited disease characterized by pathognomonic histologic features such as dilation of glomerular capillaries with huge lipoprotein-rich materials called lipoprotein thrombi. Although type III HLP is generally recognized in typical LPG, histologic alterations 


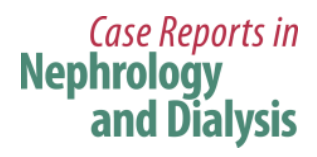

Case Rep Nephrol Dial 2015;5:204-212

\begin{tabular}{l|l}
\hline DOI: 10.1159/000441715 & ○ 2015 The Author(s). Published by S. Karger AG, Basel
\end{tabular} www.karger.com/cnd

Takasaki et al.: Macrophage Infiltration into the Glomeruli in Lipoprotein Glomerulopathy

characteristic of renal lipidosis, e.g., foam cells, sclerosis, and vacuoles, are rarely seen. Furthermore, infiltration of foamy macrophages in the glomeruli is usually absent [2]. To date, 15 novel apoE variants associated with LPG have been identified, and the histological and clinical manifestations of LPG have been considered to be common among patients with different apoE variants [2]. In the present case, LPG was diagnosed based on characteristic lipoprotein profiles and the presence of apoE Tokyo/Maebashi mutation. However, a variety of changes in ordinary renal lipidosis were also noticeable; to the best of our knowledge, this is the first report of LPG showing so many macrophages in the glomeruli.

Although apoE mutation has been recognized as playing a principal role in the pathogenesis of LPG, some other factors are inferred to be present in the pathogenesis of LPG, because many asymptomatic carriers of apoE variants are recognized [2, 7]. In an animal model, Kanamaru et al. [8] found LPG-like glomerular lesions induced by the chronic graftversus-host reaction in $\mathrm{Fc} \gamma$ receptor ( $\mathrm{FcR} \gamma$ )-deficient mice. Furthermore, Ito et al. [9] generated LPG-like changes in apoE and FcR $\gamma$ double-knockout mice by injecting various apoE vectors. These results suggest that macrophage impairment may be one of the mechanisms responsible for the development of lipoprotein thrombi and the absence of macrophages in LPG. From this point of view, other etiological factors besides apoE mutations related to LPG may play an important role in the coexistence of lipoprotein thrombi and foamy macrophages within the same glomerulus in the present case.

On the other hand, several cases of apoE2 homozygote-related glomerulopathy (apoE2GN) showing marked infiltration of foamy macrophages and sclerosis have been reported $[10,11]$. Although apoE2 has extremely reduced binding affinity for LDL receptors, very few apoE2 homozygote cases develop type III HLP and apoE2-GN. It is generally believed that apoE2 homozygote cases develop type III HLP and apoE2-GN, when some genetic, hormonal and environmental factors are added to apoE2 mutation [12]. Actually, most previously reported cases of apoE2-GN were associated with diabetic nephropathy. Because there is no evidence of diabetes in the present case, it is possible that mutated apoE related to LPG might play a role as an additional factor in the development of apoE2-GN-like lesions.

To the best of our knowledge, three cases of LPG with apoE2 allele have been reported [13-15]. Kinomura et al. [13] and Cautero et al. [14] demonstrated the cases of homozygous E2/E2 with heterozygous apoE single mutation, Arg150Gly (apoE Okayama) and Arg150Cys (apoE Modena), respectively. These two cases showed the distinctive pathological findings of LPG and neither documented the presence of foamy macrophages in the glomeruli. ApoE Tokyo/Maebashi mutation is a 9-bp deletion (3-amino acid deletion), whereas all other apoE variants related to LPG are a single-point mutation. The coexistence of this unique apoE related to LPG and classical apoE2 might be the leading cause of the specific renal pathology with lipoprotein thrombi and foamy macrophages. On the other hand, one of the 7 patients reported in the case series of LPG from China [15] seemed to carry the combination of heterozygous apoE Tokyo/Maebashi and heterozygous apoE2 alleles and show the typical renal pathology of LPG. The combination of apoE genotypes was likely to be same as our case; however, the detailed results of apoE DNA analysis were not illustrated, and serum apoE phenotype $(E 2 / 3)$ is different from that of our case (E1/2), although the reason for this is not clear. An accumulation of more cases of LPG with apoE2 allele is required to elucidate the mechanism of development of LPG and the relationship between macrophage and renal lipidosis.

In the first biopsy of the present case, CD68+ foam cells infiltrated some part of the apoE-positive region within the glomerulus, but did not exist in lipoprotein thrombi despite apoE positivity. These results suggest that in type III HLP occurring with these two apoE mutations, macrophages could recognize apoE2 and infiltrate into the glomerulus to clear 
Takasaki et al.: Macrophage Infiltration into the Glomeruli in Lipoprotein Glomerulopathy

lipids including apoE2, whereas they might not be capable of recognizing apoE derived from LPG-related apoE gene, resulting in an accumulation of lipids in the extracellular area and the development of lipoprotein thrombi. The histopathological findings in the present case suggest that properties of apoE are crucial in the development of LPG rather than macrophage impairment.

In conclusion, this is the first report of a case of LPG with marked foamy macrophage infiltration in the glomerulus. After bezafibrate treatment, not only lipoprotein thrombi but also foamy macrophages decreased. The patient was a compound heterozygote for a novel mutation of apoE associated with LPG and classical apoE2. It is intriguing that the combination of genes related to LPG and familial type III HLP expresses renal histology characteristics of both diseases simultaneously. Furthermore, this case seems to provide important clues to understanding the involvement of macrophages in the pathogenesis of LPG and apoE2-GN.

\section{Statement of Ethics}

The study of the patient was approved by the institutional ethics committee of Saiseikai Yamagata Saisei Hospital, and written informed consent was obtained from the patient before commencement of the phenotype and gene analysis.

\section{Disclosure Statement}

The authors declare that there are no conflicts of interests regarding the publication of this paper.

\section{References}

1 Saito T, Sato H, Kudo K, Oikawa S, Shibata T, Hara Y, Yoshinaga K, Sakaguchi H: Lipoprotein glomerulopathy: glomerular lipoprotein thrombi in a patient with hyperlipoproteinemia. Am J Kidney Dis 1989;13:148-153.

$\longrightarrow 2$ Saito T, Matsunaga A, Ito K, Nakashima H: Topics in lipoprotein glomerulopathy: an overview. Clin Exp Nephrol 2014;18:214-217.

-3 Oikawa S, Matsunaga A, Saito T, Sato H, Seki T, Hoshi K, Hayasaka K, Kotake H, Midorikawa H, Sekikawa A, Hara S, Abe K, Toyota T, Jingami H, Nakamura H, Sasaki J: Apolipoprotein E Sendai (arginine 145->proline): a new variant associated with lipoprotein glomerulopathy. J Am Soc Nephrol 1997;8:820-823.

-4 Matsunaga A, Sasaki J, Komatsu T, Kanatsu K, Tsuji E, Moriyama K, Koga T, Arakawa K, Oikawa S, Saito T, Kita T, Doi T: A novel apolipoprotein E mutation, E2 (Arg25Cys), in lipoprotein glomerulopathy. Kidney Int 1999;56:421-427.

-5 Konishi K, Saruta T, Kuramochi S, Oikawa S, Saito T, Han H, Matsunaga A, Sasaki J: Association of novel 3amino acid deletion mutation of apolipoprotein E (Apo E Tokyo) with lipoprotein glomerulopathy. Nephron 2014;83:214-218.

6 Ogawa T, Maruyama K, Hattori H, Arai H, Kondoh I, Egashira T, Watanabe T, Kobayashi Y, Morikawa A: A new variant of apolipoprotein E (apo E Maebashi) in lipoprotein glomerulopathy. Pediatr Nephrol 2000;14:149-151.

7 Saito T, Sato H, Oikawa S, Kudo K, Kurihara I, Nakayama K, Abe K, Yoshinaga K, Sakaguchi H: Lipoprotein glomerulopathy: report of a normolipidemic case and review of the literature. Am J Nephrol 1993;13:64-68.

-8 Kanamaru Y, Nakao A, Shirato I, Okumura K, Ogawa H, Tomino Y, Ra C: Chronic graft-versus-host autoimmune disease in Fc receptor $\gamma$ chain-deficient mice results in lipoprotein glomerulopathy. J Am Soc Nephrol 2002;13:1527-1533.

-9 Ito K, Nakashima H, Watanabe M, Ishimura A, Miyahara Y, Abe Y, Yasuno T, Ifuku M, Sasatomi Y, Saito T: Macrophage impairment produced by Fc receptor gamma deficiency plays a principal role in the development of lipoprotein glomerulopathy in concert with apoE abnormalities. Nephrol Dial Transplant 2012;27:3899-3907. 
Takasaki et al.: Macrophage Infiltration into the Glomeruli in Lipoprotein Glomerulopathy

10 Kawanishi K, Sawada A, Ochi A, Moriyama T, Mitobe M, Mochizuki T, Honda K, Oda H, Nishikawa T, Nitta K: Glomerulopathy with homozygous apolipoprotein E: a report of three cases and review of the literature. Case Rep Nephrol Urol 2013;3:128-135.

-11 Suzaki K, Kobori S, Ueno S, Uehara M, Kayashima T, Takeda H, Fukuda S, Takahashi K, Nakamura N, Uzawa $\mathrm{H}$, et al: Effects of plasmapheresis on familial type III hyperlipoproteinemia associated with glomerular lipidosis, nephrotic syndrome and diabetes mellitus. Atherosclerosis 1990;80:181-189.

12 Mahley RW, Huang Y, Rall SC Jr: Pathogenesis of type III hyperlipoproteinemia (dysbetalipoproteinemia). Questions, quandaries, and paradoxes. J Lipid Res 1999;40:1933-1949.

13 Kinomura M, Sugiyama H, Saito T, Matsunaga A, Sada KE, Kanzaki M, Takazawa Y, Maeshima Y, Yanai H, Makino H: A novel variant apolipoprotein E Okayama in a patient with lipoprotein glomerulopathy. Nephrol Dial Transplant 2008;23:751-756.

14 Cautero N, Di Benedetto F, De Ruvo N, Montalti R, Guerrini GP, Ballarin R, Spaggiari M, Smerieri N, De Blasiis MG, Rompianesi G, Iemmolo RM, Marino M, Bertolotti M, Zivieri S, Gerunda GE: Novel genetic mutation in apolipoprotein E2 homozygosis and its implication in organ donation: a case report. Transplant Proc 2010;42:1349-1351.

15 Han J, Pan Y, Chen Y, Li X, Xing G, Shi J, Hou P, Zhang H, Wang H: Common apolipoprotein E gene mutations contribute to lipoprotein glomerulopathy in China. Nephron Clin Pract 2010;114:c260-c267. 


\section{Case Reports in \\ Nephrology and Dialysis}

Takasaki et al.: Macrophage Infiltration into the Glomeruli in Lipoprotein Glomerulopathy

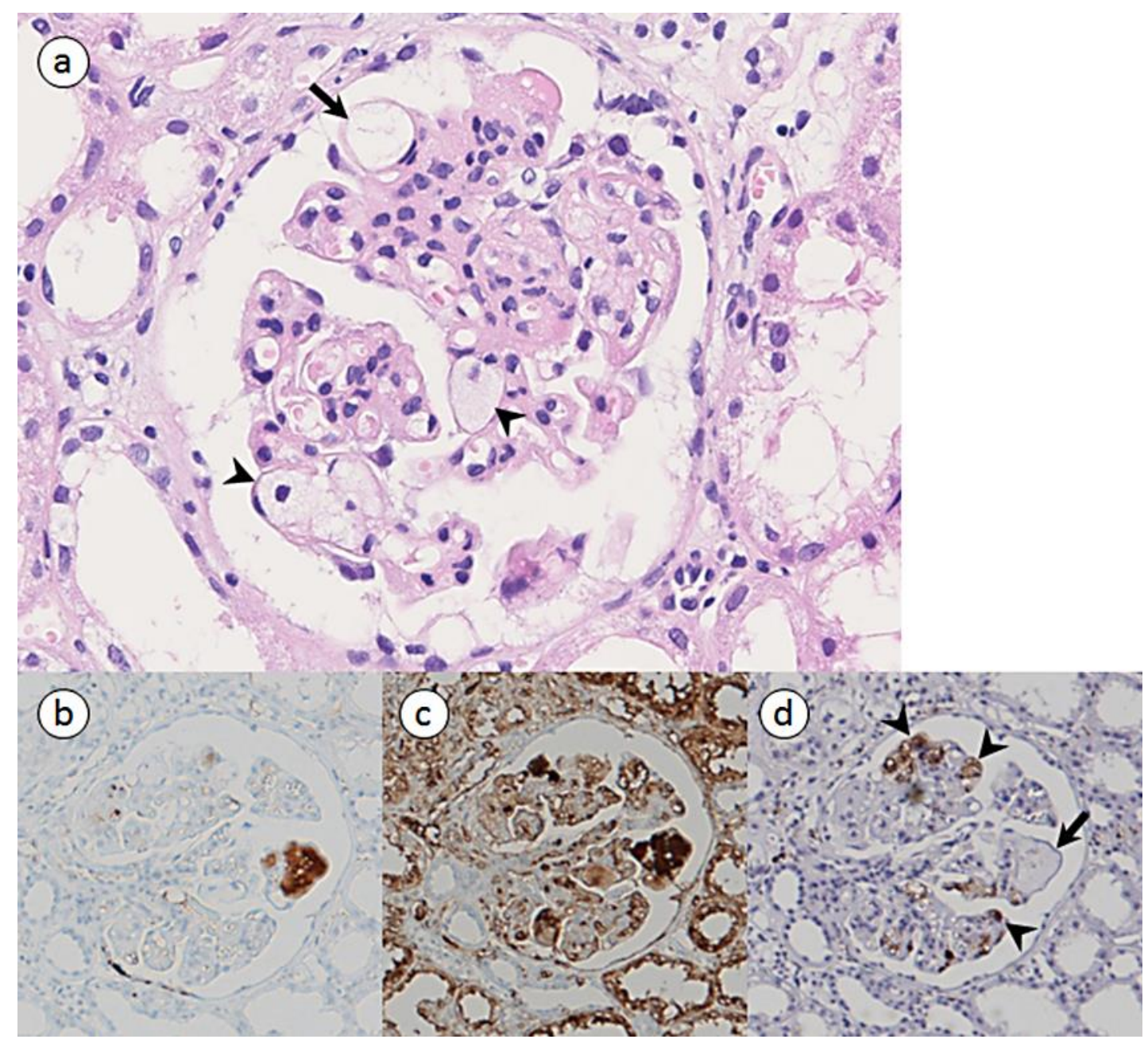

Fig. 1. Light microscopic findings of the first biopsy. a Lipoprotein thrombi (arrow) and foam cells (arrowhead) are observed within the same glomerulus. b-d Immunohistochemical studies using serial sections: Strong staining for both apoB (b) and apoE (c) is observed at lipoprotein thrombi, and positivity for apoE is also observed at sites other than the lipoprotein thrombi within the glomeruli. $\mathbf{d}$ CD68+ foam cells (arrowheads) have infiltrated in the apoE-positive part other than lipoprotein thrombi (arrow). 


\section{Case Reports in \\ Nephrology and Dialysis}

Takasaki et al.: Macrophage Infiltration into the Glomeruli in Lipoprotein Glomerulopathy
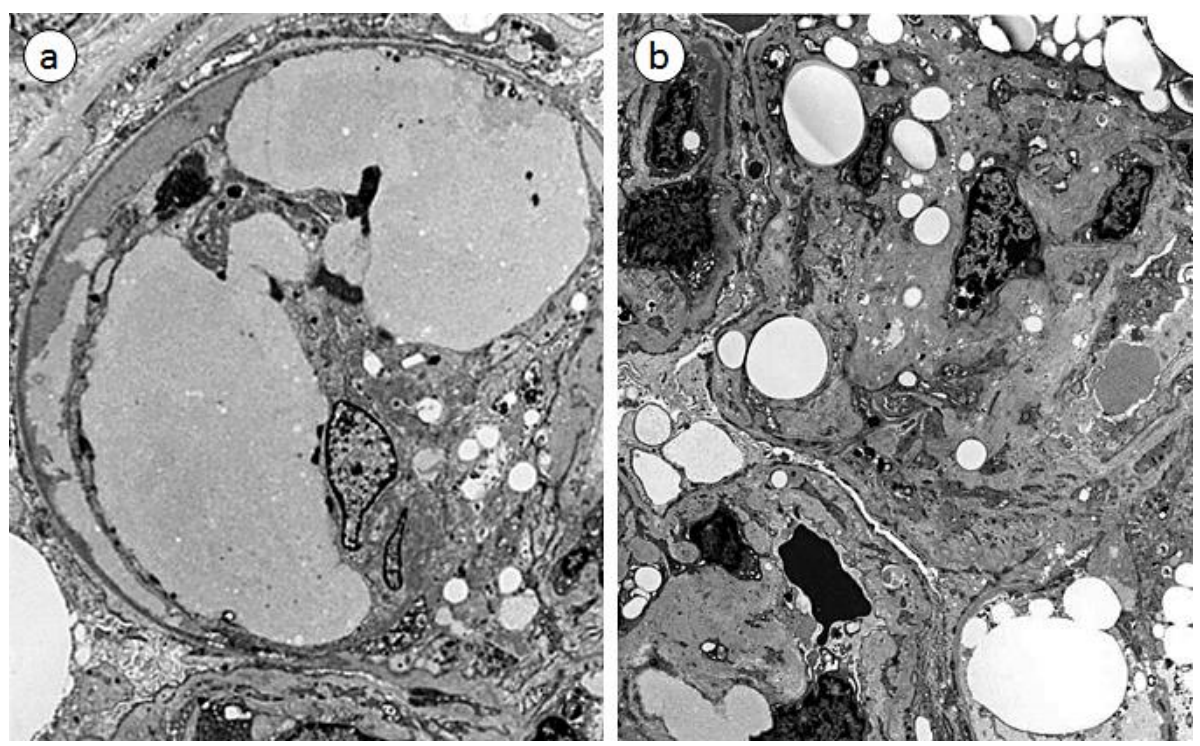

Fig. 2. Electron microscopic findings of the first biopsy. Intra-capillary lipoprotein thrombus-like substances (a) and foamy changes (b) are observed. 
Takasaki et al:: Macrophage Infiltration into the Glomeruli in Lipoprotein Glomerulopathy
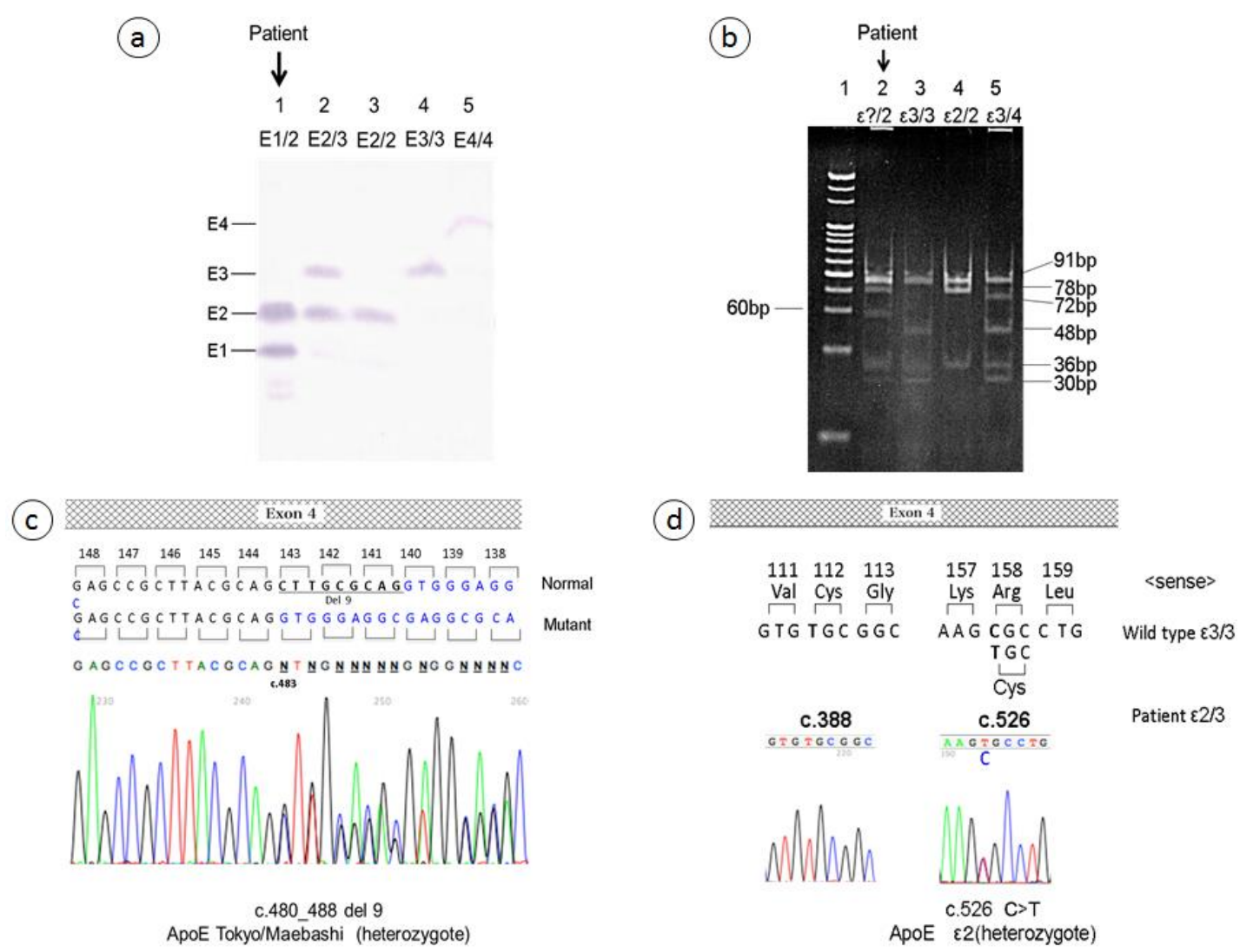

Fig. 3. a ApoE phenotype determined by isoelectric focusing polyacrylamide gel electrophoresis (IEF). Lane 1, apoE1/2 (patient); lane 2, apoE2/3; lane 3, apoE2/2; lane 4, apoE3/3 (wild type); lane 5, apoE4/4. b ApoE genotype examined by restriction fragment length polymorphism (RFLP). PCR-amplified DNA of the apoE gene, including codons 112 and 158, was digested with HhaI. Lane 1, DNA size marker; lane 2, patient; lane 3, $\varepsilon 3 / 3$; lane 4, $\varepsilon 2 / 2$; lane 5, $\varepsilon 3 / 4$. In lane 2, the 91-, 78-, and 36-bp fragments which are consistent with $\varepsilon 2$, are observed. Moreover, there are also 30 -bp and around 60-bp fragments that are not observed in $\varepsilon 2$, $\varepsilon 3$, and $\varepsilon 4$. $\mathbf{c}$, d Sequence analysis of PCR-amplified DNA of the apoE gene. c A 9-bp deletion (3-amino acid deletion) in codon 141 to codon 143 of the apoE gene is observed. There are overlaps of nucleotide peaks before codon 144, indicating that the apoE alleles are heterozygous. $\mathbf{d}$ Both the sequence CGC (arginine; wild type) and the sequence TGC (cysteine) are observed in codon 158 of the apoE gene, indicating that the apoE2 (Arg158Cys) alleles are heterozygous. 